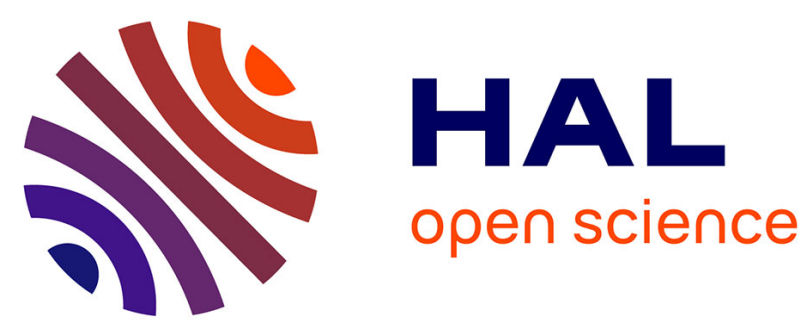

\title{
Les circulants entre métropoles européennes à l'épreuve de leurs mobilités: une lecture temporelle, spatiale et sociale de la pénibilité
}

Hadrien Dubucs, Françoise Dureau, Matthieu Giroud, Christophe Imbert, Isabelle I. André-Poyaud, Françoise Bahoken

\section{To cite this version:}

Hadrien Dubucs, Françoise Dureau, Matthieu Giroud, Christophe Imbert, Isabelle I. André-Poyaud, et al. Les circulants entre métropoles européennes à l'épreuve de leurs mobilités : une lecture temporelle, spatiale et sociale de la pénibilité. Articulo - Journal of Urban Research, 2011, 7, 15p. hal-00994278

\section{HAL Id: hal-00994278 \\ https://hal.science/hal-00994278}

Submitted on 28 May 2014

HAL is a multi-disciplinary open access archive for the deposit and dissemination of scientific research documents, whether they are published or not. The documents may come from teaching and research institutions in France or abroad, or from public or private research centers.
L'archive ouverte pluridisciplinaire HAL, est destinée au dépôt et à la diffusion de documents scientifiques de niveau recherche, publiés ou non, émanant des établissements d'enseignement et de recherche français ou étrangers, des laboratoires publics ou privés. 


\title{
Articulo - Journal of Urban Research
}

$7 \mid 2011$ :

Tiring mobilities

\section{Les circulants entre métropoles européennes à l'épreuve de leurs mobilités. Une lecture temporelle, spatiale et sociale de la pénibilité}

Circulating between European cities: A temporal, spatial and social reading of arduous mobility

\author{
HADRIEN Dubucs, Françoise Dureau, MatTHIEU Giroud, \\ CHRISTOPHE IMBERT, ISABELLE ANDRÉ-POYAUd AND FRANÇOISE \\ BAHOKEN
}

\begin{abstract}
s
Français English

Cet article interroge les circulations d'individus entre Lisbonne et quatre autres métropoles européennes (Berlin, Bruxelles, Londres, Paris) sous l'angle de la pénibilité. L'approche proposée articule trois dimensions, à savoir la construction des pratiques de circulation entre métropoles à l'échelle biographique, le fonctionnement familial induit par la circulation et l'expérience des lieux fréquentés au quotidien dans chacune des métropoles. La prise en compte de ces trois dimensions permet de caractériser les circulants en termes de situations de mobilité à travers lesquelles est envisagée la pénibilité. Les situations de mobilité bien vécues semblent traduire un relatif équilibre dans les modes de vie associés aux circulations. L'article met cependant en évidence une série de facteurs qui peuvent contribuer à fragiliser, ou à nuancer ces situations : la lassitude, l'absence d'espace d'identification et de projet, la gestion de l'absence du circulant au sein de la famille, la modification du rapport à des lieux de ressourcement, la limitation des pratiques dans les lieux de la circulation. Au total, il n'existe pas de segmentation claire entre ces situations de mobilité du point de vue de la pénibilité. Des situations de mobilité très diverses peuvent être vécues de manière différente. La pénibilité dépend finalement d'une combinaison de facteurs qui, pris isolément, n'ont pas d'effet mécanique.
\end{abstract}

This article examines the circulation of people between Lisbon and four other European cities (Berlin, Brussels, London, Paris) in terms of the difficulties that it can cause. The proposed approach articulates three dimensions, namely the construction of practices of circulation between cities at a biographical scale, the way families deal with the circulation of one or more of its members, and the daily experience of places frequented in each of the cities. Taking into account these three dimensions offers a means to characterize the people who circulate in 
terms of mobility situations through which they conceive of the wearisome dimension of circulation. Mobility situations that are well appreciated seem to reveal a relatively balanced way of life associated with circulation. However, the article identifies a number of factors that may contribute to weaken or qualify these situations: weariness, lack of space of identification and project, management within family of the absence of those who circulate, changes in relation to places of healing, and limitations in practicing the places that structure the space of circulation. Overall, there is no clear segmentation between these situations of mobility in terms of difficulty. Situations of mobility that are very different can be experienced in many varied ways. The tiring dimension of circulation ultimately depends on a combination of factors which, taken separately, have no mechanical effect.

\section{Index terms}

Mots-clés : circulation, Lisbonne, métropole européenne, mobilités, pénibilité

Keywords : arduous mobility, circulation, European metropolis, Lisbon, mobility

\section{Full text}

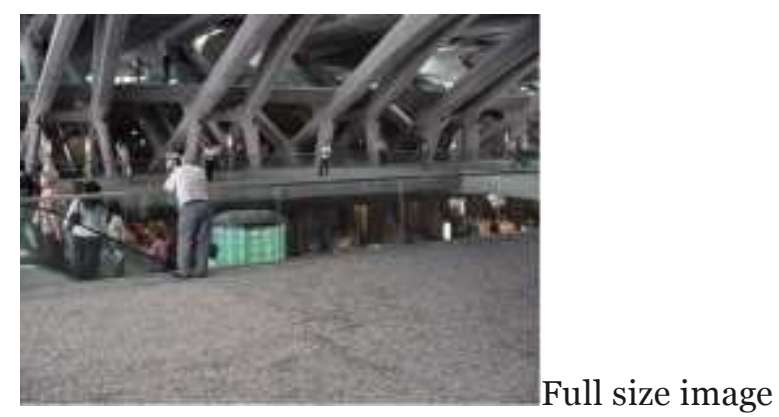

Credits : (C) Matthieu Giroud

\section{Introduction}

Cet article vise à interroger les circulations entre grandes métropoles européennes sous l'angle de la pénibilité. Il s'appuie sur les données d'enquêtes réalisées dans le cadre du programme Mobilités circulaires entre les métropoles européennes et reconfigurations des espaces de vie (MEREV), financé par l'Agence nationale de la recherche et mené entre 2007 et 2010 par Géographie-cités (UMR 8504 Universités Paris 1 et Paris 7 - CNRS) et par Migrinter (UMR 6588 Université de Poitiers - CNRS). Cette recherche a pour objectif de saisir la manière dont les populations qui réalisent des déplacements réguliers et/ou fréquents entre métropoles européennes à partir d'un lieu de résidence principale situé à Berlin, Bruxelles, Lisbonne, Londres ou Paris, reconfigurent leurs espaces de vie tant du point de vue des pratiques que de leurs représentations.

Notre positionnement repose sur la définition de la circulation donnée par Zelinsky (1971 : 225-226) : elle renvoie à une grande variété de déplacements habituellement de courte durée, répétitifs ou cycliques, mais qui ont tous en commun l'absence de toute intention déclarée d'un changement de résidence permanent ou durable. Il s'agit de considérer toutes les formes de la circulation entre villes européennes qui peut concerner une grande diversité de profils individuels et reposer sur des motifs variés (professionnel, familial, de loisirs). Ce travail s'inscrit en outre au sein d'une approche globale des mobilités qui d'une part insiste sur le caractère intégré et indissociable des différentes formes de mobilités (Zelinsky 1971, Bassand et Brulhardt 1980, Courgeau 1988, Brun 1993, Bassand et Kaufmann 2000, Lévy et Dureau 2002) et d'autre part privilégie une lecture biographique des mobilités et des lieux fréquentés, éclairant la dynamique de constitution des espaces de vie (Courgeau 1988, GRAB 1999). 
Dans cette perspective nous postulons que la pénibilité des circulations n'est compréhensible qu'en considérant trois de leurs dimensions - temporelle, spatiale et sociale - articulées les unes aux autres :

4 - La construction des pratiques de circulation entre métropoles européennes à l'échelle biographique : les pratiques de circulation peuvent être parfois appréhendées comme des constructions réalisées sur le temps long à l'échelle de la vie de l'individu. On peut alors se demander si l'existence ou non de cette épaisseur biographique dans la mise en place de la circulation agit sur le sentiment de pénibilité de l'expérience circulatoire. A-t-elle notamment une influence sur la faculté des individus à s'attacher aux lieux de la circulation, à savoir jouer sur la complémentarité des lieux pratiqués au quotidien, ajuster ou reproduire certaines habitudes ici ou là, ou encore sur les compétences pratiques mises en œuvre dans la mobilité ? Dans la logique de cette lecture dynamique de la pénibilité de l'expérience circulatoire, la manière dont les circulants se projettent dans l'avenir doit être aussi prise en compte.

- Les pratiques de circulation entre métropoles européennes et le fonctionnement familial : l'approche biographique proposée amène à questionner les effets des pratiques de circulation sur le fonctionnement du ménage et inversement le rôle de l'entourage dans la réalisation de la circulation. Il s'agit de montrer que la nature des arrangements et des arbitrages réalisés au sein du ménage ou de l'entourage pour satisfaire d'un côté la vie familiale et domestique, de l'autre la vie professionnelle, est un des facteurs importants du sentiment de pénibilité de l'expérience circulatoire.

$6 \quad$ - Les pratiques de circulation et l'expérience des lieux fréquentés au quotidien dans chacune des métropoles européennes : la troisième dimension constitutive de notre approche est de considérer la pénibilité de l'expérience circulatoire au regard des activités réalisées dans chacun des lieux (et notamment chacune des métropoles) de l'espace de la circulation et dans les temps du déplacement lui-même. Il s'agit ici de dépasser une lecture de la circulation qui ne porterait que sur la mobilité entre les grandes métropoles ainsi que sur les motifs annoncés de fréquentation, pour envisager plus concrètement ce qui se joue dans et entre chacune des métropoles fréquentées, notamment en matière de pratiques et d'accès aux ressources urbaines localisées.

$7 \quad$ La prise en compte de ces trois dimensions permet de caractériser les circulants en termes de situations de mobilité à travers lesquelles est envisagée la pénibilité. Par situation de mobilité, on entend un mode de vie caractéristique d'un certain moment de la trajectoire familiale, professionnelle et personnelle de l'individu, organisé sur plusieurs lieux et impliquant des circulations entre eux. Les situations de mobilité sont donc évolutives et inégalement stables au long de la vie d'un individu, mais aussi d'un individu à l'autre. La prise en compte de situations de mobilité invite aussi à dépasser le strict échelon individuel en tenant compte des pratiques de mobilité et de circulation des membres de la famille de l'individu considéré et à saisir leurs effets réciproques.

8 Cet article présente les résultats d'enquêtes effectuées à Lisbonne et réalisées en trois temps. Une première phase consistait à rechercher des circulants dans des lieux susceptibles d'en accueillir comme les aéroports, les gares ferroviaires et routières. Le questionnaire utilisé auprès de 600 personnes a porté sur les déplacements effectués en dehors de la métropole de résidence au cours de l'année écoulée.

9 La seconde phase de l'enquête a été réalisée auprès de 60 individus dont 40 identifiés comme " circulants ». A été considéré comme " circulant » tout individu ayant séjourné au moins deux fois dans un même lieu hors de son agglomération de résidence au cours des douze mois précédant l'enquête. Le positionnement adopté consiste ainsi à ne définir le circulant que par la pratique d'une circulation : il s'agit 
donc d'une catégorie qui ne coïncide pas nécessairement avec les catégorisations socioprofessionnelles habituelles. Cette seconde phase visait à recueillir au sein d'un autre questionnaire la biographie résidentielle, professionnelle et « de circulation » (lieux fréquentés de façon régulière et intensive) des personnes interrogées ; à décrire de façon plus précise la circulation entre les métropoles sur la dernière année (conditions de logement, lieux pratiqués dans les différentes métropoles et en dehors de ces métropoles) ; à identifier les lieux de résidence actuels de la famille proche (parents, conjoints, enfants).

Enfin, 16 des 40 circulants ayant répondu à ce second questionnaire ont participé à des entretiens semi-directifs lors d'une troisième phase. Le critère de sélection de ces 16 personnes a été celui de la plus grande diversité de profils de circulants révélés par les deux phases précédentes (nombre, durée, motifs et destination des séjours). Les objectifs de ces entretiens étaient d'étudier les représentations de la circulation : origine, contraintes des déplacements pour la personne interrogée et son entourage ; vécu des déplacements et évolution de la pratique des lieux (habitudes, processus de repli, d'ouverture) ; projets dans l'avenir proche (maintien ou non de la circulation).

Cet article repose sur les résultats des deuxième et troisième phases de ce travail d'enquête, précisément sur le contenu thématique des entretiens. Les résultats présentés ici sont en effet issus d'analyses thématiques (application systématique d'une grille d'analyse) qui ont porté sur les différents registres permettant de définir les situations de mobilité - l'apprentissage de la circulation, la pratique de la circulation entre les villes européennes, la pratique de ces villes européennes - et sur les projets de vie à l'horizon de 2-3 ans. Nous avons veillé, lors de l'exploitation des résultats, à croiser le plus possible les cas étudiés via les entretiens avec les catégories plus générales, identifiées dans la seconde phase de l'enquête, auxquelles ils se réfèrent.

La pénibilité circulatoire n'apparait presque jamais de manière explicite dans les entretiens : ce thème n'était pas au cœur de l'enquête et l'on peut supposer une certaine difficulté à exprimer spontanément cette pénibilité dans le contexte actuel de valorisation de la mobilité. Pour autant, il se révèle en creux lorsqu'on identifie les situations de mobilité, en mettant en relation les trois dimensions évoquées plus haut. Un des révélateurs de la pénibilité réside notamment dans l'épaisseur biographique et dans la capacité de projection de la situation de mobilité observée. Notre hypothèse est en effet que la pénibilité se révèle à partir du moment où un individu ne souhaite plus maintenir sa situation de mobilité, engagée depuis plusieurs années ou non, telle qu'elle est. Nous considérons que la volonté que peut avoir un individu de modifier dans un avenir plus ou moins proche une situation de mobilité (la difficulté à inscrire une situation de mobilité dans une dynamique et un projet personnel et familial) est l'expression d'une insatisfaction, d'un inconfort, d'une lassitude voire plus profondément d'un certain mal de vivre. Nous rejoignons ainsi les approches qui considèrent tout individu comme un acteur de son existence, capable avec certes plus ou moins d'intensité et d'autonomie face à des contraintes extérieures, d'ajuster - même de manière minimale - certaines de ses pratiques sociales et spatiales pour améliorer son mode de vie. Nous défendons donc une approche de la pénibilité qui considère que ce sentiment résulte d'une multiplicité de facteurs : il n'y a pas de relation mécanique entre une forme de mobilité (rythme, intensité) et une manière de la vivre.

\section{Des situations de mobilité bien vécues: diversité des profils et complexité des facteurs}


Deux situations de mobilité bien vécues ressortent des entretiens : la première correspond à des cadres d'entreprise circulant très intensément entre métropoles européennes et mondiales, la seconde renvoie à des individus circulant très régulièrement en Europe mais pour des motifs familiaux. Les individus concernés sont très différents par leurs caractéristiques individuelles (situation familiale, position socioprofessionnelle, âge, rythmes et lieux de la mobilité). Mais ils partagent le fait de " bien vivre » leur situation de mobilité, ce qu'indique notamment leur volonté explicite de ne pas la remettre en question à court ou moyen terme, voire de la pérenniser. Ce « bien vivre » partagé s'explique par la conjonction de plusieurs facteurs qui relèvent des trois dimensions déjà évoquées.

\section{Bien vivre le déplacement}

On sait qu'une partie de la pénibilité créée par la mobilité est liée au temps du déplacement lui-même (voyage en train ou en avion, temps passé dans la ville de séjour). C'est celui-ci qui entraîne notamment chez certains cadres internationaux une lassitude physique et morale : fatigue et stress du voyage, lever matinal pour prendre l'avion (Chevrier et Sauvage 2006). Les entretiens réalisés montrent que certains de ces cadres livrent au contraire un discours positif sur leurs mobilités. C'est notamment le cas de Paulo, 41 ans, responsable de la filiale portugaise d'une multinationale du secteur de l'enfance, qui vit à Lisbonne avec sa femme et leur enfant et réalise des déplacements très fréquents entre certaines villes européennes (Amsterdam, Barcelone, Paris), ainsi qu'entre l'Europe et le siège new-yorkais de l'entreprise. C'est également le cas de Joao, 36 ans, cadre dirigeant d'une multinationale américaine, vivant à Londres avec sa femme et leurs trois enfants et qui effectue sur un circuit comparable environ quatre voyages internationaux par semaine depuis deux ans.

15 Plusieurs facteurs peuvent expliquer le fait que la situation de mobilité apparaît globalement bien vécue. Dans un contexte de fluidité accrue des déplacements permise par les infrastructures de transport (multiplication des liaisons aériennes, accessibilité des aéroports) et les conditions administratives du voyage intraeuropéen (possibilités d'enregistrement par internet ou sur des bornes), ces circulants font preuve d'une réelle compétence à la circulation : connaissance des horaires, des lieux de réunion et de destination, bonne évaluation des temps disponibles et des temps de trajet, capacité à voyager sans valise, doublement des effets personnels, etc. La circulation est parfois facilitée par des employeurs, qui devant les fortes contraintes temporelles des déplacements mettent en place des dispositifs d'accompagnement de la mobilité tels que des taxis réservés qui assurent les liaisons entre l'aéroport et l'hôtel ou des réservations préalables des billets d'avion et des chambres d'hôtel. Les compétences individuelles sont aussi liées à l'ancienneté dans la circulation professionnelle : Paulo voyage ainsi depuis 12 ans et Joao depuis 6 ans.

16 L'ancienneté dans la circulation peut être créatrice de réseaux de connaissances sur place, comme en témoigne Joao : «Je profite [de mes déplacements à Paris et à Rotterdam] pour joindre des personnes que je connais. C'est possible par le fait que je suis dans l'entreprise depuis beaucoup d'années ». L'existence de "points de chute » et de sociabilités personnelles apporte ainsi un certain agrément au déplacement. De manière plus générale, l'ancienneté de l'expérience circulatoire permet à certains de ces cadres d'entreprise de créer et de maintenir des habitudes, qui traduisent alors une capacité à investir et à s'approprier chacune des métropoles fréquentées. Joao par exemple choisit toujours les mêmes hôtels dans les villes où il se rend. A Barcelone, il a privilégié la proximité d'un parc où il peut faire du footing tôt le matin avant sa journée de travail. On trouve ainsi l'idée de se ménager des « temps pour soi » : footing matinal, se lever plus tôt le matin pour profiter de la 
ville. Le rythme effréné des séjours professionnels est évoqué avec un certain regret par les circulants professionnels. Ils s'efforcent de " voler » certains moments à leurs agendas très contraints, qu'ils mettent à profit pour des activités dans des lieux identifiés selon des critères plus personnels et affinitaires : magasin de bandes dessinées, dîner chez des amis sur place. Ces «temps pour soi » sont mobilisés soit en prolongeant des habitudes associées au lieu de résidence, soit sur le mode de la complémentarité avec les habitudes de consommation dans le lieu de résidence habituel.

La capacité à bien vivre le déplacement se retrouve aussi chez des personnes qui ne sont pas des circulants professionnels, aux rythmes de circulation nettement moins soutenus et qui ne bénéficient pas d'un encadrement par l'employeur. C'est le cas de Lisboètes séjournant régulièrement chez les membres de leur famille proche résidant dans d'autres villes européennes. Les circulations d'échelle européenne fondées sur les visites familiales se retrouvent fréquemment dans l'échantillon des 40 circulants identifiés dans l'enquête. Parmi les 16 personnes ayant fait l'objet d'entretiens approfondis, plusieurs exemples illustrent cette situation : Manuela, veuve retraitée de 64 ans qui réside à Lisbonne et se rend plusieurs fois par an à Londres où sa fille réside et aux Açores ; Suzana, retraitée de 74 ans, qui voyage en Europe et rend régulièrement visite à son fils à Londres ; Ivone, étudiante angolaise de 23 ans, qui réside à Sintra près de Lisbonne et qui rend visite plusieurs fois par an à ses oncles et tantes en France (à Tarbes, Nantes et Paris) et à Zurich où habitent sa mère et ses frères.

Les cas inhabituels de Manuela et Suzana sont particulièrement intéressants, car ils illustrent une situation de mobilité bien vécue par des individus âgés. Or, tout laisserait à penser que, pour cette catégorie de personnes, les circulations internationales tendraient à être limitées à des occasions exceptionnelles. L'exemple des beaux-parents de Rosa (dont la situation sera présentée plus loin) apparaît à cet égard beaucoup plus évident : pour pouvoir bénéficier de leur soutien pratique au quotidien à partir de sa seconde grossesse, Rosa est obligée de mettre un terme à sa situation de bi-résidence entre Londres et Lisbonne et de se sédentariser dans cette dernière ville. En effet, les difficultés que ses parents et beaux-parents éprouvent à se déplacer empêchent de mettre en place une circulation régulière avec Londres.

Plusieurs facteurs interviennent dans la facilité qu'éprouvent Manuela, Suzana et Ivone à effectuer plusieurs fois par an leurs visites familiales. C'est tout d'abord l'ancienneté de la pratique du voyage : Ivone effectue ses visites familiales en Europe depuis le début de l'adolescence tandis que Manuela et Suzana ont exercé une activité professionnelle et ont fait des voyages d'agrément à l'étranger très tôt par rapport aux femmes portugaises de leur génération (au début de ses études pour Suzana, et de sa vie active pour Manuela). Dans le cas de Manuela, le goût des voyages répond d'ailleurs clairement à une enfance vécue comme beaucoup trop sédentaire et géographiquement bornée, dans l'île de Terceira aux Açores. Ensuite, Suzana et Manuela entretiennent un rapport de grande familiarité avec Londres. Elles connaissaient en effet bien la ville avant que leurs enfants ne s'y installent. Leur grande autonomie et leur connaissance de Londres acquise sur le temps long contribuent à leur assurer une relation très intime avec l'espace londonien : la ville est un lieu pratiqué (flâneries, événements culturels, sociabilités) et représenté de manière très précise.

En outre, l'ancienneté de l'expérience personnelle du voyage rend le déplacement beaucoup plus facile à vivre du point de vue de sa perception : la représentation prend en compte l'évolution des moyens de transport. Par exemple, Suzana affirme être frappée par la facilité du voyage entre Lisbonne et Londres aujourd'hui, par rapport aux années 1960 : elle réserve ses billets et fait son enregistrement par internet et trouve que l'accès depuis l'aéroport au centre-ville de Londres est devenu très aisé. Enfin, pour ces enquêtées, le déplacement c'est déjà le voyage. Elles ne 
décrivent leurs déplacements qu'en termes très positifs. Le temps des préparatifs peut certes être perçu comme laborieux, mais le temps du déplacement est associé au voyage et à la découverte : "De sorte que ce stress de préparer des bagages [...] de penser à tout... je disais toujours que l'heure bénie est l'heure du check in : ce qui a été oublié n'a plus d'importance, on ne peut plus aller le chercher! » (Manuela).

\section{La famille, prétexte et soutien à la mobilité}

Dans toutes ces situations, les relations entre les individus et leurs familles sont un facteur décisif de la non-pénibilité de leurs mobilités. Le motif familial lui-même joue dans la satisfaction associée à la mobilité et contribue à la pérennisation de la situation de mobilité.

Dans les cas d'Ivone, Suzana et Manuela, la présence de famille à l'étranger offre des opportunités de voyages d'agrément. La mise à profit touristique de la présence de l'entourage à l'étranger peut constituer une habitude durable : Manuela suit sa fille lorsqu'elle se rend en voyage professionnel à Dubaï et en vacances en République dominicaine ; Suzana suivait son fils lorsque, doctorant, il participait à des colloques. Ivone profite de ses séjours à Paris pour fréquenter les commerces africains, s'acheter des vêtements et flâner dans la ville avec ses cousines : "On fait des promenades, on voit des monuments. On va aussi dans des restaurants, pour la gastronomie Paris c'est bien. Et puis c'est bien aussi pour la musique, pas tellement française, mais africaine », dit-elle.

De plus, le fait d'avoir un " point de chute » familial facilite très clairement ces déplacements, sur le plan financier comme en matière d'organisation matérielle. Mais la famille à l'étranger ne représente pas seulement des points de chute pour des voyages : les relations familiales constituent souvent de puissants motifs d'une circulation migratoire (Doraï, Hily et Loyer 1998) et sont constitutives de systèmes résidentiels familiaux (Bonnin et de Villanova 1999, Dureau 2006). Dans les mobilités pour motifs familiaux, le rapport au temps est souvent moins contraint que dans le cadre d'une circulation professionnelle. Les circulations ne sont pas décrites comme des absences répétées, mais comme une manière d'être présent auprès des membres de la famille. La mobilité est ici un moyen de contribuer à la cohésion du groupe familial d'origine. Manuela et Suzana partagent ainsi leur vie entre Lisbonne, Londres et leur lieu d'origine (Açores pour Manuela et Torres Novas au Portugal pour Suzana). Dans chacun des lieux, elles fournissent une aide à leur famille : Suzana s'occupe de ses petits-enfants à Torres Novas (lieu de rassemblement de la famille, pour les vacances ou les fêtes) et à Londres ; Manuela module ses séjours pour être là où sa présence est jugée la plus nécessaire pour ses proches. Elle s'occupe de sa mère très âgée lorsqu'elle se rend plusieurs mois par an aux Açores ; à Lisbonne, elle s'occupe de ses petits-enfants à la sortie de l'école ; à Londres, elle rend visite à sa fille. Manuela et Suzana habitent donc trois lieux à la fois, s'y déplacent de façon autonome, y aménagent des espaces d'agrément et entretiennent avec chaque lieu un rapport intime, ce qu'on ne saurait comprendre sans tenir compte de l'expérience des voyages accumulée tout au long de leur vie adulte. Dans le cas d'Ivone, les circulations permettent d'entretenir des relations fortes avec les membres de sa famille élargie, tout en maintenant avec celle-ci une distance qu'elle juge nécessaire. Signe que ces mobilités sont bien vécues, Ivone préfère rester à Lisbonne alors que sa famille l'incite régulièrement à s'installer en France où les opportunités professionnelles seraient plus nombreuses. Il s'agit là encore d'un fonctionnement stable pour l'instant, qui combine l'agrément des visites en Europe avec la liberté que lui procure cet éloignement de l'entourage.

Les situations de mobilité bien vécues rassemblent donc des individus très différents par leur âge, leur catégorie socioprofessionnelle, leur situation professionnelle, ainsi que le rythme et l'intensité de leurs mobilités. Dans la 
non-pénibilité des circulations, aucun facteur explicatif unique ni même dominant ne se distingue. Les indicateurs de cette non-pénibilité (stabilité temporelle des situations de mobilité, éléments de discours) mêlent étroitement trois dimensions. La première est biographique et se rapporte à l'ancienneté de la circulation créatrice de compétences personnelles, aux ressources mobilisables pour faciliter la circulation et à l'encadrement professionnel. La seconde concerne les rapports pratiques et idéels aux lieux mis en relation par ces circulations, tels que le développement d'attachement aux lieux, les usages à des fins personnelles et les sociabilités. La troisième a trait aux relations familiales : hébergement chez des membres de la famille, recours à la mobilité pour trouver une "bonne distance » avec la famille, arbitrages au sein du couple. A plusieurs égards, les relations familiales tendent à favoriser les circulations lorsque la famille est dispersée entre plusieurs lieux après des mouvements migratoires ou lorsqu'il s'agit d'apporter un soutien à un parent laissé seul par le conjoint circulant. On retrouve là le rôle de la famille-entourage souligné notamment par Bonvalet et Lelièvre (1995) avec, peut-être, une spécificité du contexte portugais. Ces observations plaident également en faveur de la prise en compte d'une famille qui ne se réduit pas à la famille nucléaire.

\section{Entre mobilité bien vécue et mobilité éprouvante}

Les situations décrites précédemment semblent traduire un relatif équilibre dans les modes de vie associés aux circulations, vécues par les individus concernés sans que domine l'expression d'une expérience pénible. L'analyse approfondie de l'ensemble du corpus met cependant en évidence une série de facteurs qui peuvent contribuer à fragiliser ou à nuancer ces situations d'équilibre.

\section{Quand la mobilité peut devenir éprouvante : projets flous et lassitude}

L'un des apports majeurs de l'approche biographique a été de montrer qu'un événement ou une suite d'événements peuvent reconfigurer l'espace de vie d'un individu : un divorce, la perte d'un emploi, le passage à la retraite, etc. (Courgeau et Lelièvre 1989). Les recherches sur le passage à l'âge adulte (Calvès et al. 2006) en sont un bon exemple : cette situation perçue comme transitoire jusqu'à l'acquisition de l'autonomie résidentielle, l'insertion professionnelle et la formation d'un couple est susceptible, dans la durée, de devenir usante. L'expérience pendant l'enfance et l'adolescence de situations de mobilité (séjours longs et répétés) peut ainsi s'avérer contraignante au moment où il s'agit de " s'installer » (Meyfroidt 2002). Le thème des projets à moyen terme abordé dans les entretiens donne l'occasion de comprendre cette lassitude.

Prenons l'exemple de José : ce fils d'ambassadeur a une grande habitude des voyages liés aux différentes affectations de son père et à un nomadisme étudiant. Résidant aujourd'hui à Lisbonne où il n'a pas d'attaches familiales, il occupe sans grand enthousiasme un travail d'assistant comptable. Pour lui, la mobilité est devenue une contrainte, source de fatigue et de malaise, car il ne dispose pas véritablement d'espace de référence, d'identification (Gotman 1993), ni donc de projet :

« Je ne saurais pas bien répondre à cette question [Où vous voyez-vous dans deux ans ?], c'est une chose qui me poursuit tous les jours. Je m'étais donné quatre ans 
pour changer de vie et je n'ai pas réussi... c'est un martyre psychologique [...] Comment je vois l'avenir ? Je ne sais pas, je peux seulement dire ce que j’aimerais faire. J'aimerais beaucoup à l'avenir en termes généraux avoir une famille, être père. [...] En termes professionnels, j'aimerais faire quelque chose, maintenant quoi je ne sais pas, mais je suis en train de réfléchir à cela. Je suis en train d'essayer de comprendre... Je pense que si je suis aussi confus c'est dû à mon parcours, je trouve que je me suis trop dispersé,je ne sais pas, c'est confus dans ma tête. »

En dépit d'une grande habitude de la mobilité, José est insatisfait de sa situation actuelle. La mobilité, que José a jusqu'alors plutôt subie, représente une contrainte à la réalisation de ses aspirations, certes encore confuses, à plus de stabilité et de sédentarité. Il est intéressant de mettre en regard ce témoignage avec celui de Joao, dont le cas a été évoqué plus haut. En effet, Joao vit bien sa situation de mobilité alors que, contrairement à José, celle-ci est extrêmement intense, ne s'inscrit pas dans une tradition familiale en la matière et pèse lourdement sur l'organisation du ménage. A la différence de José, Joao a la capacité de définir sa situation de mobilité comme une étape qui prend sens dans sa trajectoire professionnelle et familiale. Ce mode de vie n'est perçu que comme temporaire, Joao partageant avec son épouse le projet d'une réinstallation à Lisbonne, qui fait ainsi figure à la fois d'espace de référence, d'identification et de projet. En attendant, il importe pour le couple de rester le plus proche possible au quotidien des autres membres de la famille, grâce aux technologies de télécommunication.

Ces deux exemples montrent qu'il n'existe pas de lien simple entre pénibilité et ancienneté d'une situation de mobilité intense. Par ailleurs, pour peu que l'hypermobilité assure un maintien des retours réguliers vers un lieu d'ancrage fort, celle-ci peut-être bien vécue. On est ici au cœur de la dialectique entre mobilité et ancrage (Hirschhorn et Berthelot 1996). Pour José, la mobilité qui n’a cessé depuis son enfance freine sa recherche d'ancrage, alors que pour Joao c'est la mobilité qui lui permet de maintenir son ancrage à Lisbonne et d'envisager un retour à moyen ou long terme. Cela étant, l'équilibre trouvé par Joao pourrait se trouver remis en cause si son projet résidentiel devait être contrarié. A l'inverse, si José parvenait à s'ancrer en un lieu, son rapport à la mobilité deviendrait vraisemblablement moins problématique. On remarque d'ailleurs dans plusieurs cas que la non pénibilité de certaines situations de mobilité est associée à l'existence de lieux de ressource(ment) familiaux et plus globalement sociaux et identitaires.

\section{Des lieux de ressource(ment) pour les hypermobiles : un facteur d'équilibre?}

La présence d'une maison de famille, à Lisbonne ou dans d'autres régions portugaises, permet de se retrouver, de se ressourcer, de mettre temporairement de côté un mode de vie fatigant. Dès lors, les contraintes et les limitations de l'accès à de tels lieux, parfois imposées par la situation de mobilité, peuvent être mal appréciées et ainsi contribuer à produire un sentiment de pénibilité.

Pour Antonio par exemple, un banquier marié et père de deux enfants qui se rend plusieurs fois par an à Bruxelles dans le cadre de son travail, la possession d'une maison située dans l'Alvor semble déterminante dans son mode de vie. Cette maison est un véritable prolongement de sa résidence de la banlieue de Lisbonne (Almada). Il s'y rend environ une fois par mois depuis une quinzaine d'années, y retrouve des amis dont certains résident eux-mêmes à proximité de sa résidence principale. Il y va avec épouse et enfants, ainsi que de temps en temps avec son père natif de la région. Ses enfants adolescents y ont des amis d'enfance et commencent désormais à fréquenter seuls la maison. Antonio est très attaché à cette maison tout comme il l'est à Lisbonne. Tant que sa mobilité dans les villes européennes ne 
contrarie pas son rapport à ces deux lieux, on peut supposer qu'il continuera à bien vivre cette situation. L'équilibre reste toutefois fragile, comme l'illustre Leticia, une autre enquêtée. Cette chercheuse lisboète est très attachée à l'habitude de passer un week-end sur deux et toutes ses vacances dans son village d'origine. Elle y retrouve famille et amis. Elle vit donc mal les séjours estivaux d'un mois et demi à Paris que lui imposent ses recherches post-doctorales. Ceux-ci sont une source de mal être et de frustration croissante.

Lorsque la circulation dans un cadre professionnel est bien vécue en dépit de la fatigue qu'elle engendre, il n'y a pas nécessairement de repli ou d'enfermement sur le domicile conjugal. Au contraire, d'autres déplacements réguliers peuvent se produire vers des résidences secondaires et ces déplacements ont une fonction de ressourcement. Il est difficile de savoir à quel point les situations évoquées sont à replacer dans un contexte portugais où l'institution familiale joue un rôle régulateur important. Cependant la diversité des modes de fonctionnement étudiés au prisme de situations de forte mobilité d'un ou de plusieurs membres permet d'éviter l'écueil d'une vision trop monolithique de la famille portugaise. Par ailleurs, l'existence de lieux d'ancrage familiaux qui forment l'ossature de l'espace de vie ne signifie pas un enracinement des personnes (Imbert 2005) : nombreuses sont les situations où la circulation et l'attachement à plusieurs lieux s'inscrivent dans des régimes stables. Sans qu'il soit possible d'approfondir cette question avec le matériau recueilli, on peut supposer que le recours à des lieux considérés comme des ressources hors de la résidence habituelle contribue à rendre l'hypermobilité plus supportable même s'il engendre des déplacements supplémentaires.

\section{Gérer l'absence d'un proche : de l'individu à la famille élargie}

Si une situation de mobilité telle que la pendularité de longue distance ou la plurirésidence peut être bien gérée par la personne mobile, ses absences répétées peuvent toutefois rendre la vie difficile à son entourage. Ces difficultés ont été relevées à de nombreuses reprises pour le fonctionnement du couple (Bonnet, Collet et Maurines 2006, Legrand et Ortar 2008). La famille élargie apparaît alors comme un acteur fondamental pour pallier l'absence d'un conjoint, en particulier pour certains moments de la vie, tels que les grossesses et les naissances.

C'est ce qu'illustre le cas de Rosa et de son mari, qui ont partagé pendant deux ans une expérience de bi-résidence entre Lisbonne et Londres. Au moment de l'entretien, alors enceinte de son second enfant, Rosa a choisi depuis plusieurs mois de résider de nouveau à Lisbonne. Rosa témoigne de l'importance de la proximité de ses beaux-parents et de sa mère dans ce choix : ils se montrent très disponibles pour la soutenir quotidiennement ou pour garder leur petite-fille quand Rosa décide de se rendre à Londres pour le travail ou pour des week-ends avec son mari. Dans cette situation, c'est la carrière du mari qui a été privilégiée : même si elle maintient une activité de consultant en informatique depuis son domicile, Rosa a abandonné une carrière d'informaticienne à Amsterdam pour se consacrer à ses enfants. Elle considère toutefois cette situation comme temporaire car à aucun moment il n'est question de s'installer à Londres et l'avenir du couple et des enfants n'est envisagé qu'à Lisbonne. Si le soutien familial parvient à atténuer certains effets de l'absence du mari, celle-ci reste en définitive problématique - elle génère semble-t-il de la solitude et affecte les relations sociales - et source de tensions.

L'absence d'un conjoint est en effet souvent plus pesante pour un adulte que celle d'un parent ou d'un enfant. Elle se heurte à des normes du fonctionnement du couple en termes de cohabitation et de relation amoureuse. Elle entraîne aussi des contraintes matérielles, concernant le partage des tâches domestiques ou 
l'éducation des enfants. Au-delà de cette considération, il apparaît que l'entourage est diversement armé pour gérer cette absence.

\section{Un effet tunnel ? Insatisfaction dans la pratique de la ville de séjour et dans les conditions de déplacement}

Parfois l'impossibilité d'avoir une pratique souhaitée du lieu de destination entraîne une certaine frustration concernant les voyages professionnels.

C'est le cas de Carlos, homme divorcé de 44 ans, père de deux enfants, formateur au sein d'un réseau international d'écoles de police. Il regrette aujourd'hui de ne pouvoir profiter davantage de ses déplacements professionnels. Dans le cadre de son activité, il effectue en effet depuis cinq ans un séjour mensuel de trois jours dans une ville européenne : Düsseldorf, Florence, Londres, Nicosie, Oslo, Prague. Le quartier général de ce réseau se trouve dans la grande couronne de la banlieue londonienne, où il s'est rendu trois fois en 2009. Carlos exprime sa frustration quant à l'extrême encadrement des modalités du voyage et du séjour : tout est planifié depuis l'aéroport. Les participants restent ensemble dans le centre de congrès ou la caserne souvent situés en périphérie d'agglomération (comme à Londres) et n'ont que quelques heures de temps libre chaque jour. S'ils visitent le centre-ville, il s'agit d'une visite en groupe des hauts-lieux de la ville suivie d'un dîner de gala. A cette grégarité de tous les instants s'ajoute le caractère formel des relations entre collègues où il s'agit de rester en représentation :

"Si je vais à une réunion, à une formation ou à un congrès, ou à toute autre occasion, je suis obligé d'avoir une tenue correcte, je suis dans un cadre social formel avec mes collègues internationaux. On ne peut jamais se détendre alors que quand on part en vacances c'est différent, n'est-ce pas ? Quand on est en vacances, on porte une tenue décontractée, on fait ce que l'on veut, on a le temps de se poser. Au début, c'est toujours sympa, mais à la longue c'est fatigant. Parfois, on en a assez des aéroports... mais je pense quand même que c'est bien, c'est très bien de mener cette vie. »

L'évocation par Carlos de sa pratique des villes européennes suggère une forme d'effet tunnel qu'il déplore d'autant plus qu'il apprécie les city-trips touristiques. De temps en temps, il lui arrive d'ailleurs de prolonger son séjour le week-end avec sa compagne venue le rejoindre pour visiter Prague ou parce qu'il est invité par un collègue chypriote avec qui il a noué des relations amicales. Les occasions sont rares puisque les séjours se font la plupart du temps en début de semaine. Même s'il exprime une relative satisfaction à l'égard de sa situation de mobilité, Carlos souhaite toutefois à moyen terme obtenir une mutation à Porto pour se rapprocher de sa compagne qui réside à Braga.

La frustration liée à l'encadrement des séjours professionnels est un élément récurrent dans les discours de tous les cadres mobiles. Du point de vue de la manière de vivre la mobilité, les dispositifs d'encadrement apparaissent donc ambivalents. Ils facilitent le déplacement, mais ils limitent aussi la nature et les lieux des pratiques effectuées pendant les séjours. Cependant, tout ne paraît pas si simple : Paulo, dont le cas a été abordé précédemment, rappelle par exemple que les entreprises ont eu tendance il y a quelques années à imposer à leurs cadres de rester le week-end dans la ville de séjour pour bénéficier des meilleurs tarifs des billets d'avion. Cet allongement des durées de séjour a été alors perçu de manière contrastée : il permettait de moins contraindre dans le temps la tenue des réunions et de profiter du lieu de destination, mais il augmentait aussi le temps passé hors du domicile familial. 
forces, révélé par la situation de mobilité, entre l'entreprise et ses employés, le pouvoir exercé par l'employeur à travers l'injonction d'écourter ou au contraire de prolonger les séjours de travail. De telles limitations prennent une toute autre dimension quand elles s'inscrivent au sein d'un contexte marqué par une intensification des déplacements professionnels et par la recherche de réduction des coûts des séjours. Cet effort de réduction des coûts conduit ainsi de plus en plus les entreprises à opter pour des compagnies low cost ou à cibler certains horaires plus contraignants ; il détermine aussi en partie l'ordre des différentes étapes du circuit ainsi que la durée des séjours. Dans un autre ordre d'idées, le renforcement et la multiplication des dispositifs de sécurité dans les aéroports, en contraignant la fluidité des déplacements, peuvent aussi contribuer à modifier la perception et la pratique de la mobilité de ces circulants. Les conditions structurelles et techniques de la mobilité jouent un rôle majeur dans la pénibilité des situations de mobilité. Des évolutions structurelles des conditions de déplacement sont en effet susceptibles de transformer le rapport à la mobilité. Elles peuvent modifier l'organisation des déplacements et même provoquer des déménagements lorsque de nouvelles opportunités ou contraintes émergent.

\section{Conclusion}

Les situations de mobilité que connaissent les circulants étudiés apparaissent au final très hétérogènes. Elles sont évolutives et inégalement stables : certaines connaissent une forte stabilité, d'autres sont recomposées par un événement familial ou professionnel. Elles sont en outre complexes par leur configuration spatiale, la présence ou non de lieu(x) d'ancrage dans la durée et leurs motifs souvent entremêlés. Il n'existe pas de segmentation claire entre elles du point de vue de la pénibilité. Des situations de mobilité très diverses peuvent être vécues de manière négative. On n'observe aucun lien univoque entre, par exemple, l'intensité des circulations et la pénibilité. La pénibilité dépend finalement d'une combinaison de facteurs qui, pris isolément, n'ont pas d'effet mécanique. On a vu l'ambivalence des dispositifs d'encadrement des déplacements et des séjours : ils facilitent le voyage et en même temps limitent les activités qui peuvent lui être associées. Ces facteurs relèvent des trois dimensions familiale, biographique et spatiale envisagées au départ.

Est-on pour autant condamné à ne décrire qu'une collection de situations individuelles de mobilité, dans lesquelles la pénibilité est vécue de façon particulière ? On pourrait par exemple penser que le genre est une caractéristique différenciant fortement les individus du point de vue de la pénibilité de leurs mobilités, notamment parce que les femmes participent de façon plus importante à la gestion des enfants (Ortar 2008). Notre échantillon ne permet pas de comparer le degré de pénibilité selon le genre à situation de mobilité comparable. En revanche, parmi les 600 enquêtés de la première phase on observe une spécialisation par genre du type de mobilité : les hommes sont très majoritaires parmi les enquêtés qui effectuent des circulations fréquentes et impliquant un grand nombre de lieux. Les femmes effectuent quant à elles plus fréquemment des circulations pour motifs familiaux. Ces observations suggèrent que le genre n'intervient donc pas tant dans la pénibilité de la mobilité que dans la forme prise par la mobilité elle-même, qui résulte d'arbitrages en amont au sein du couple et de la famille élargie.

De manière plus hypothétique, il nous semble qu'un dénominateur commun à toutes les situations analysées est que l'absence d'ancrage spatial rend difficilement supportable une situation de mobilités fréquentes. Réciproquement, si elle perturbe la pratique d'un lieu d'ancrage, la situation de mobilités tend à être difficilement vécue. L'ancrage spatial se traduit par les pratiques, l'appropriation, voire 
l'attachement ou l'identification. De nombreux travaux en sciences sociales abordent directement la question de l'ancrage spatial et soulignent que celui-ci ne contredit nullement le développement de pratiques de mobilité. La question plus particulière des mobilités éprouvantes ne fait ici que confirmer l'étroitesse du lien entre mobilité et ancrage. Ce lien mérite d'autant plus d'être analysé que les discours des individus questionnés sur leurs mobilités et sur la manière dont ils la vivent tendent à être influencés par un contexte de valorisation de la mobilité comme capacité à s'émanciper d'attaches territoriales.

\section{Bibliography}

DOI are automaticaly added to references by Bilbo, OpenEdition's Bibliographic Annotation Tool.

Users of institutions which have subscribed to one of OpenEdition freemium programs can download references for which Bilbo found a DOI in standard formats using the buttons available on the right.

Format

APA

MLA

Chicago

The Bibliographic Export Service is accessible via institutions subscribing to one OpenEdition freemium programs. If you wish your institution to become a subscriber to one OpenEdition freemium programs and thus benefit from our services, please write to:contact@openedition.org

Bassand M, Brulhardt MC. 1980. Mobilité spatiale. Saint-Saphorin, Georgi.

Bassand M, Kaufmann V. 200o. Mobilité spatiale et processus de métropolisation : quelles interactions?, in Bonnet M, Desjeux D. (eds) Les territoires de la mobilité. Paris, PUF: 129-140.

Bonnet E, Collet B, Maurines B. 2006. Mobilités de travail, dissociation spatio-temporelle et carrières familiales, in Bonnet $\mathrm{M}$, Aubertel $\mathrm{P}$. (eds) La ville aux limites de la mobilité. Paris, PUF: $183-192$.

Bonnin P, de Villanova R. (eds) 1999. D’une maison l'autre. Parcours et mobilités résidentielles. Paris, Creaphis.

Format

APA

MLA

Chicago

The Bibliographic Export Service is accessible via institutions subscribing to one OpenEdition freemium programs. If you wish your institution to become a subscriber to one OpenEdition freemium programs and thus benefit from our services, please write to:contact@openedition.org

Bonvalet C, Lelièvre E. 1995. Du concept de ménage à celui d'entourage : une redéfinition de l'espace familial. Sociologie et Sociétés 27(2): 177-190.

DOI : $10.7202 / 001076$ ar

Brun J. 1993. La mobilité résidentielle et les sciences sociales. Transfert de concept et questions de méthodes. Les Annales de la Recherche Urbaine 59-60: 3-14.

Calvès $\mathrm{AE}$, Bozon $\mathrm{M}$, Diagne $\mathrm{A}$, Kuépé $\mathrm{M}$. 2006. Le passage à l'âge adulte : repenser la définition et l'analyse des " premières fois ", in Antoine P, Lelièvre E. (eds) Etats flous et trajectoires complexes. Observation, modélisation, interprétation. Paris, INED-CEPED: 137-156.

Chevrier S, Sauvage A. 2006. Les cadres internationaux à l'épreuve du déplacement, in Bonnet M, Aubertel P. (eds) La ville aux limites de la mobilité. Paris, PUF: 175-182.

Format

APA

MLA

Chicago

The Bibliographic Export Service is accessible via institutions subscribing to one OpenEdition freemium programs. If you wish your institution to become a subscriber to one OpenEdition freemium programs and thus benefit from our services, please write to:contact@openedition.org

Courgeau D. 1988. Méthodes de mesure de la mobilité spatiale. Migration interne, mobilité temporaire, navettes. Paris, Ined.

DOI : $10.2307 / 1533496$ 
Format

APA

MLA

Chicago

The Bibliographic Export Service is accessible via institutions subscribing to one OpenEdition freemium programs. If you wish your institution to become a subscriber to one OpenEdition freemium programs and thus benefit from our services, please write to:contact@openedition.org

Courgeau D, Lelièvre E. 1989. Analyse démographique des biographies. Paris, Ined.

DOI : $10.2307 / 1533444$

Doraï K, Hily M.-A, Loyer F. 1998. Bilan des travaux sur la circulation migratoire, Rapport final au Ministère de la Solidarité et de l'Emploi, Direction de la Population et des Migrations. Poitiers, Migrinter.

Dureau F. 2006. L'observation des systèmes résidentiels dans les villes colombiennes, in Dureau F, Golaz V. (eds) Mobilité et résidence. Paris, CEPED.

Gotman A. 1999. Géographies familiales, migrations et générations, in Bonvalet C, Gotman A, Grafmeyer Y. (eds) La famille et ses proches : l'aménagement des territoires. Paris, INED-PUF: 69-133.

GRAB (Groupe de Réflexion sur l'Approche Biographique). 1999. Biographies d'enquête. Bilan de 14 collectes biographiques. Paris, Ined-PUF.

Hirschhorn M, Berthelot J.-M. (eds) 1996. Mobilités et ancrages. Vers un nouveau mode de spatialisation? Paris, L'Harmattan.

Imbert C. 2005. Les ancrages des habitants des villes nouvelles franciliennes : des bassins de vie en construction. Paris, Université Paris 1, unpublished PhD thesis.

Legrand C, Ortar N. 2008. Caractéristiques de la surmobilité professionnelle en périurbain. Paris, Certu.

Lévy J.-P, Dureau F. (eds) 2002. L'accès à la ville. Les mobilités spatiales en question. Paris, L'Harmattan.

Ortar N. 2008. Entre ville et campagne, le difficile équilibre des périurbaines lointaines. Métropoles 3, http://metropoles.revues.org/1642 (Retrieved May 17, 2011).

Meyfroidt P. 2002. La notion d'installation dans la sociologie urbaine de langue française : émergence d'un concept. Recherches Sociologiques 33(3): 47-60.

Format

APA

MLA

Chicago

The Bibliographic Export Service is accessible via institutions subscribing to one OpenEdition freemium programs. If you wish your institution to become a subscriber to one OpenEdition freemium programs and thus benefit from our services, please write to:contact@openedition.org

Zelinsky W. 1971. The hypothesis of the mobility transition. Geographical Review 61(2): 219-219

DOI : $10.2307 / 213996$

\section{References}

Electronic reference

Hadrien Dubucs, Françoise Dureau, Matthieu Giroud, Christophe Imbert, Isabelle AndréPoyaud and Françoise Bahoken, "Les circulants entre métropoles européennes à l'épreuve de leurs mobilités. Une lecture temporelle, spatiale et sociale de la pénibilité », Articulo Journal of Urban Research [Online], 7 | 2011, Online since 19 September 2011, connection on 20 May 2014. URL : http://articulo.revues.org/1810 ; DOI : 10.4000/articulo.1810

\section{About the authors}

\section{Hadrien Dubucs}

Hadrien Dubucs is an Assistant Professor of Geography at the University of Paris 4 and a researcher at the ENeC Laboratory (UMR $8185 \mathrm{CNRS}$ ). His research deals with the relations between international migrations and intra-urban mobilities in the Paris metropolitan region. E-mail: hdubucs@gmail.com 
Françoise Dureau is a geographer and demographer. She is a Research Director at the Development Research Institute (IRD, UMR 201) in France, and a researcher at the Migrinter Laboratory (UMR 6588 CNRS), University of Poitiers. Her research interests lie in spatial mobility practices and urban change, in particular in Colombia. E-mail: f.dureau@gmail.com

\section{Matthieu Giroud}

Matthieu Giroud is an Assistant Professor of Geography at the University of ClermondFerrand. He is a researcher with the EA 997 Ceramac team and a Research Associate at the Migrinter Laboratory (UMR 6588 CNRS), University of Poitiers. His research deals with spatial mobility practices, urban change, and gentrification, notably in Lisbon. E-mail: matthieu.giroud@univ-bpclermont.fr

\section{Christophe Imbert}

Christophe Imbert is an Assistant Professor of Geography and a member of the Migrinter Laboratory (UMR 6588 CNRS) at the University of Poitiers, and at the French National Institute for Demographic Studies (INED). He specializes in mobility studies, with a particular focus on the process through which individuals, families, and populations fix territorially. E-mail: christophe.imbert@univ-poitiers.fr

\section{Isabelle André-Poyaud}

Isabelle André-Poyaud is a statistician at the Pacte Laboratory (UMR 5194), University of Grenoble and CNRS. She participates in the exploitation of survey data on daily mobilities. E-mail: isabelle.andre-poyaud@ujf-grenoble.fr

\section{Françoise Bahoken}

Françoise Bahoken is a PhD student at the Géographie-Cités Laboratory (University of Paris CNRS). She also works as a geographer at the University of Paris-Est and at the French institute of science and technology for transport, development and networks (IFSTTAR) where she participates in the processing, analysis and representation of statistical information. E-mail: frcse_bhk@yahoo.fr

\section{Copyright}

Creative Commons 3.0 - by-nc-nd, except for those images whose rights are reserved. 